\title{
Utilization pattern of kangaroo mother care after introduction in eight selected neonatal intensive care units in China
}

Xin Liu', Zhankui Li ${ }^{2}$, Xiaohui Chen ${ }^{3}$, Bei Cao ${ }^{4}$, Shaojie Yue ${ }^{5}$, Changyi Yang ${ }^{6}$, Qiongyu Liu ${ }^{7}$ Chuanzhong Yang ${ }^{8}$, Gengli Zhao ${ }^{9}$ and Qi Feng ${ }^{1 *}$ (D)

\begin{abstract}
Background: Kangaroo mother care $(\mathrm{KMC})$ is an evidence-based and cost-effective intervention that could prevent severe complications for preterm babies, however it has not been widely adopted in China. In this study, we aim to investigate the feasibility and parental experience of adopting KMC in a Chinese context by studying the implementation of a KMC program in eight self-selected neonatal intensive care units (NICUs).

Methods: A cross-sectional study of 135 preterm infants discharged from eight NICUs in April 2018. For infants information was collected on postnatal day and corrected gestational age (GA) at KMC initiation, frequency and duration of KMC provision and whether the infant was receiving respiratory support. A nurse-administered questionnaire on parents' knowledge and experience of KMC provision was administered to parents providing KMC.

Results: One hundred thirty-five preterm infants received KMC, $21.2 \%$ of all preterm infants discharged. $65.2 \%$ of those who received KMC were below 32 weeks GA, 60.7\% had a birth weight below $1500 \mathrm{~g}$, and $20.7 \%$ needed respiratory support at KMC initiation. Average KMC exposure was greater in infants born at GA $<28$ weeks that babies born at greater GA. $94.8 \%$ of parents that participated in the parental survey indicated that KMC was positively accepted by their family members; $60.4 \%$ of the parents claimed that KMC could relieve anxiety, $57.3 \%$ claimed it prompted more interactions with medical staff and 69.8\% suggested it increased parental confidence in care for their infants.
\end{abstract}

Conclusions: After advocacy, training and promotion, intermittent KMC was initiated on more immature and highrisk infants, and well-accepted by parents. We suggest continuing to promote KMC education to parents and enhancing preterm infant health.

Keywords: Preterm infants, Kangaroo mother care, Neonatal intensive care unit

* Correspondence: fengqizf@126.com

${ }^{1}$ Neonatal Intensive Care Unit, Department of Pediatrics, Peking University First Hospital, Beijing, MM, China

Full list of author information is available at the end of the article

(c) The Author(s). 2020 Open Access This article is licensed under a Creative Commons Attribution 4.0 International License, which permits use, sharing, adaptation, distribution and reproduction in any medium or format, as long as you give appropriate credit to the original author(s) and the source, provide a link to the Creative Commons licence, and indicate if changes were made. The images or other third party material in this article are included in the article's Creative Commons licence, unless indicated otherwise in a credit line to the material. If material is not included in the article's Creative Commons licence and your intended use is not permitted by statutory regulation or exceeds the permitted use, you will need to obtain permission directly from the copyright holder. To view a copy of this licence, visit http://creativecommons.org/licenses/by/4.0/ The Creative Commons Public Domain Dedication waiver (http://creativecommons.org/publicdomain/zero/1.0/) applies to the data made available in this article, unless otherwise stated in a credit line to the data. 


\section{Background}

China has sustained significant improvements in reducing child mortality, with deaths in children under the age of five falling from an estimated 18.5 per 1000 live births in 2008 to 8.6 per 1000 live births in 2018 [1]. Half of deaths in children under the age of five in China occur in the neonatal period (<28 days) and $31 \%$ of these are due to complications of prematurity [2]. China had a preterm birth rate of 6.9 per 100 live births in 2014, equating to approximately 1.2 million preterm newborns, the second highest annual number in the world [3]. These statistics show that an intervention proven to reduce mortality and morbidity in preterm newborns if adopted across China has the potential for huge beneficial impact. Kangaroo mother care (KMC) is such an intervention.

KMC originated in Bogota, Colombia in the 1970s [4]. The World Health Organization defines KMC as "care of preterm infants carried skin to skin with the mother. Its key features include continuous and prolonged skin to skin contact between the mother and the baby, and exclusive breastfeeding (ideally) or feeding with breast milk" [5]. KMC is an evidence-based and cost-effective intervention that has been demonstrated to increase newborn survival, exclusive breastfeeding, weight gain and reduce the risk of hypothermia, apnea, and serious newborn infections such as sepsis and pneumonia [6, 7]. Evidence showed that KMC increases parent-infant bonding and improves long-term psychological and intellectual development $[8,9]$. WHO recommends newborns weighing less than $2000 \mathrm{~g}$ receive continuous $\mathrm{KMC}$ when possible; and that when continuous $\mathrm{KMC}$ is not feasible, intermittent KMC should be provided based on evidence of decreased morbidity when compared to conventional care [10].

Before China's Premature Birth and Preterm Infants Intervention Program started to actively promote KMC in 2014, the policy of most neonatal intensive care units (NICUs) in China was not to allow parents into the NICUs and those that permitted parental access tended to prohibit physical contact between parent and the child [11], a practice largely in place to prevent newborn infections. A few hospitals implemented KMC to varying degrees [12-14], however it was not established as part of routine care in China and there was no standardized guideline or training. Chinese doctors and nurses had few opportunities to witness or experience the provision of KMC which hindered uptake and the development of KMC implementation strategies [15]. KMC was widely perceived by clinical staff to pose an infection risk and place an additional burden on the workload of already stretched medical staff. In addition, the space available on NICUs for KMC implementation was limited and commonly cited as a major barrier for successful initiation of KMC [16].
In 2014, the Department of Maternal and Child Health of the National Health Commission of China established the Premature Birth and Preterm Infants Intervention. The Intervention Program has worked to raise awareness and promote the implementation of $\mathrm{KMC}$ amongst its network of 50 hospitals through the use of lectures, training in the approach, and exchange experience. Ten of these hospitals volunteered to take part in a structured pilot of KMC implementation. In 2015 the hospitals were provided with 3 days theoretical and practical training in Beijing, China. In 2016 a meeting was held between representatives of these hospitals, the Premature Birth and Preterm Infants Intervention Program and external experts to draft a KMC implementation protocol for use in the pilot. Eight of these hospitals agreed to participate in implementation research to understand the feasibility and method of adoption of KMC in the context of China's NICUs. This would inform national KMC guidelines and create 'centers of excellence' to promote implementation and scale up of KMC across China. In 2017 the study design and data capture tools were developed, piloted and finalized. This cross-sectional study of KMC practice was conducted for preterm infants discharged from NICUs during the month of April 2018, it provides a snapshot of KMC implementation in participating NICU's a couple of years after the protocol had been agreed.

\section{Methods}

\section{Study design and population}

This was a cross-sectional study of infants discharged from eight NICUs in April 2018. The protocol and standard operating procedures were designed by the academic study group along with international experts, with contributions from each hospital's NICU medical and nursing staff.

The eight NICUs were self-selected from a network established under China's Premature Birth and Preterm Infants Intervention Program, these hospitals were promoting KMC and had incorporated it into their routine practice. The hospitals from seven different provinces are located in major urban cities, two are teaching hospitals and six are specialized maternal and child-health care hospitals. All are tertiary level hospitals, the number of beds in eight NICUs rang from 22 to 60 (on average 33).

Over a two-year period, staff from the eight hospitals participated in training sessions on KMC practice and management, these trainings were delivered by international and domestic experts, in addition twice yearly workshops were held to enable staffs from participating hospitals to share their experiences of $\mathrm{KMC}$ implementation.

Our study population was all preterm infants $(\mathrm{GA}<37$ weeks at birth) cared for in the eight NICUs. All preterm 
infants were discharged in April 2018, but their date of birth varied with the earliest born in January 2018. GA was determined by both early antenatal ultrasound records and clinical examination on admission to the NICU. Hospital neonatologists decided which newborns were eligible for KMC (Fig. 1). This was based on the neonatologist's perception of the balance of benefit and risk KMC posed for each newborn, dependent on their clinical condition. Provided a newborn's parents were willing, intermittent KMC could then be provided. Due to the limited availability of space and staff in all eight units, it was not possible for all eligible newborns to receive $\mathrm{KMC}$ at the same time, therefore an appointment system was implemented, with parents booking time slots for KMC provision. Newborns receiving one or more sessions of intermittent KMC during the entire hospital stay were defined as the "KMC group", others were defined as the "non KMC group". Babies not considered eligible for KMC were those deemed by physicians to have very unstable vital signs, catheters in place preventing prone KMC position, and those whose parents refused to sign informed consent; NICU preterm babies considered by clinicians to be less benefited by $\mathrm{KMC}$ (ie, "low risk" infants) were not offered KMC given the limited available NICU space for KMC beds and the aim to provide babies who would experience greater KMC benefit if safely provided. Babies who never received KMC during the entire hospital stay for mainly these reasons were defined as the non-KMC group. In brief, the non-KMC group was largely comprised of two different groups - those considered too unstable for safe $\mathrm{KMC}$; and those considered by medical staffs as "low risk" and less likely to benefit from KMC than smaller and/or sicker babies.

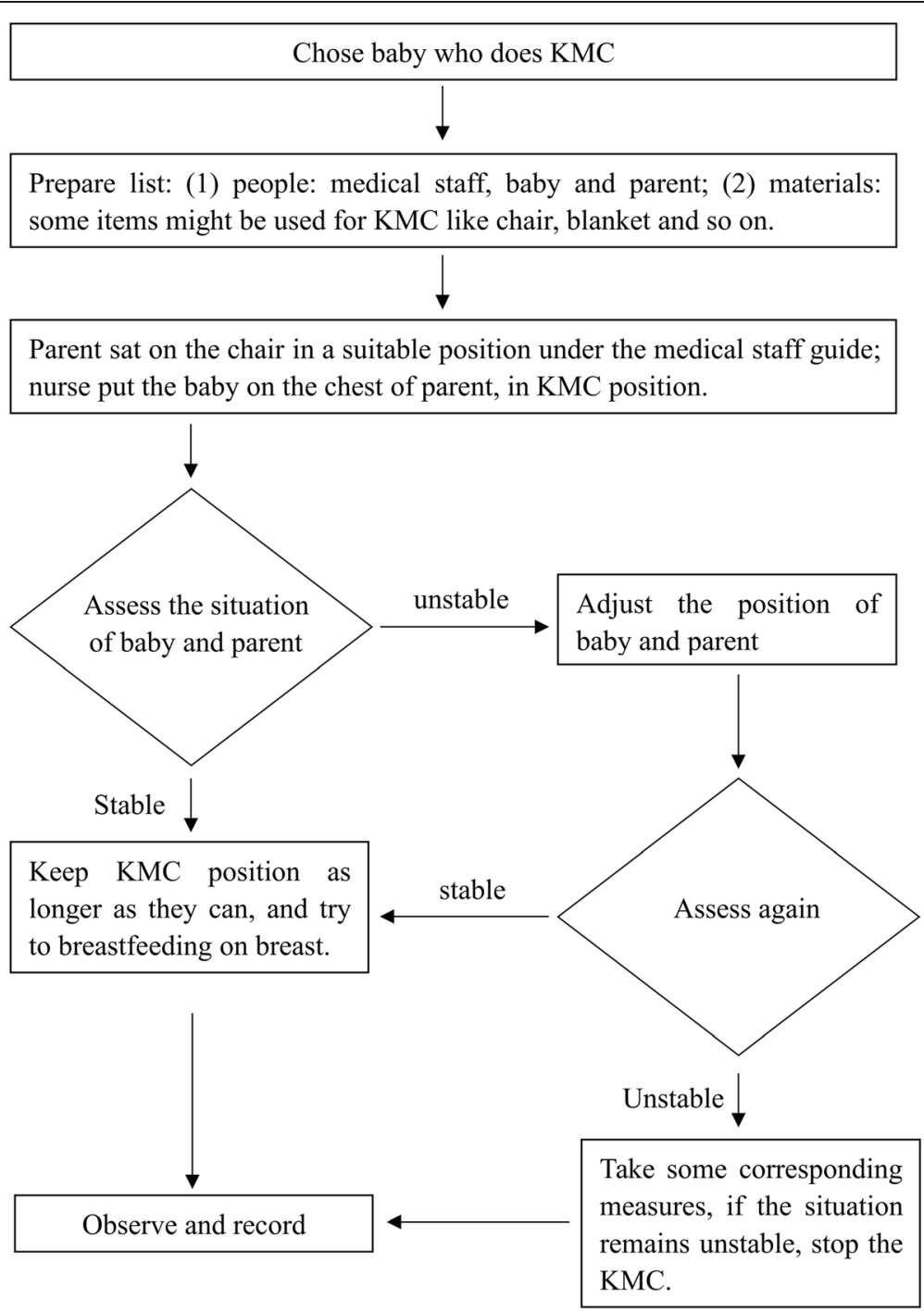

Fig. 1 Decision support flow chart of KMC implementation 
KMC was practiced using a standard protocol and flow chart (Fig. 2) developed for this KMC project, mostly adapted from international KMC practice guidance [14]. Each pilot hospital took part in the development and training of the protocol and $\mathrm{KMC}$ flow chart.

Intermittent KMC was conducted in the NICUs on a lounge chair next to the baby's incubator or bed. The baby was placed in the kangaroo position, skin to skin on the chest of its mother or father. Each KMC session last from 30 min to 2 hours, there's usually 30 time slots available per day and NICU (usually from 8:00 to 20:00). The duration of each KMC session was determined by the availability of staff and space on the NICU, the infants' condition and the parent's availability. Likewise, the number of lounge chair next to the baby's incubator or bed usually determine the number of slots available. NICUs and families were encouraged to practice KMC for longer duration and more sessions (>5) during the entire hospital stay as the project progressed, based on increasing confidence of clinical staff and adaptions made in NICU environment and practice routines.

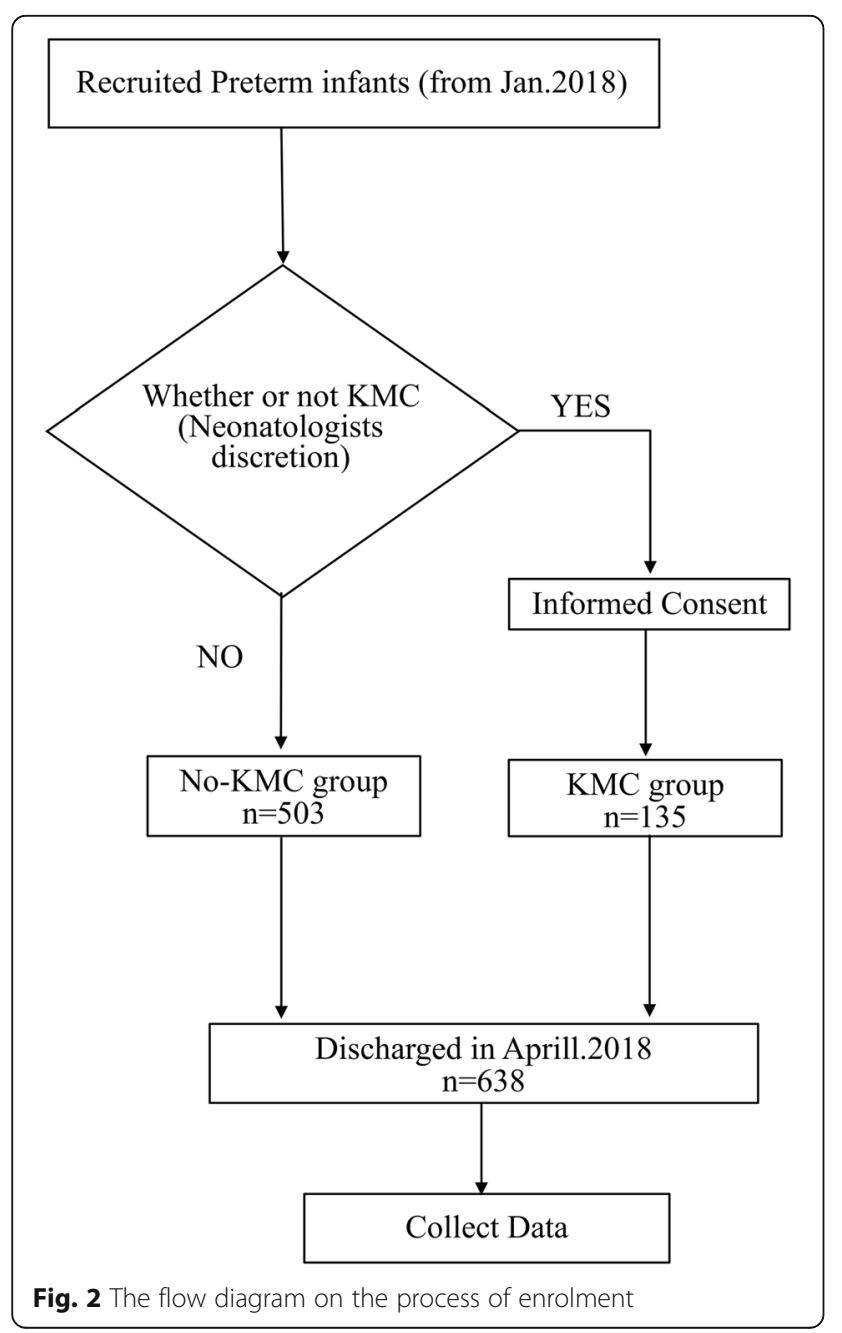

During $\mathrm{KMC}$, nursing staff were available to help and support the parents, they monitored the infants heart rate, respiratory rate and oxygen saturation levels. If the newborns condition deteriorated or emergency intervention was required, $\mathrm{KMC}$ was discontinued immediately.

\section{Measures and variables}

General information was collected from the hospital records of all discharged preterm infants whether they received KMC or not. This included GA at birth, weight on admission and discharge, and length of hospital stay. For newborns who received KMC, each KMC session was documented by a nurse using a daily recording sheet that included information on the length of the KMC session, the infant's age, gestational age (CGA), daily weight, vital signs and details of any respiratory support needed. The sheet also included the nurses name and the name of the parent providing KMC.

Parents providing KMC were surveyed after two or three KMC sessions. The survey questionnaire was administered by NICU nurses with the intention of collecting information on parents' knowledge and experience of KMC provision (See supplementary appendix). Questionnaire included closed-ended questions on KMC information (source of information), KMC experience (any assistance from medical staff, perceived benefits of $\mathrm{KMC}$, intention to continue KMC after discharge) and KMC preference (best time period, duration and frequency of KMC). We also included the following questions in the questionnaire: "Did you receive family member accept?", "How did medical staff help you during KMC?", "What are the benefits of KMC for you?"

All data was entered onto a specially designed Epidata database. A nurse from each participating NICU was identified and assumed responsibility for data entry after receiving training on data collection. Epidata data from each hospital was compiled in a central location (Peking University First Hospital) for review, reconfirmation and analysis by the authors.

Birth weight was measured using the scales available in each NICU. Respiratory support was defined as persistent oxygen via nasal cannula, invasive or noninvasive ventilation therapy. KMC exposure hours was the sum of each child's total exposure time over the course of his/her hospital stay.

\section{Statistical analysis}

Data analysis was performed using SPSS for Windows version 20 (IBM Inc., Chicago, IL). The test level was set at $\alpha=0.05$, and $p<0.05$ was considered statistically significant. Outcomes were presented for the full study population and separately for the KMC and non KMC groups. Percentage and chi-square were used to analyze subject questionnaires. Statistical significance was 
determined by chi-square, t-test or by Wilcoxon rank sum test based on the outcome of interest.

\section{Results}

\section{KMC implementation in NICUs}

KMC was conducted in NICUs of all eight pilot hospitals. Table 1 shows the characteristics of preterm infants who received and did not receive KMC. Infants born at a lower gestational age $(\mathrm{GA}<32$ weeks), with a lower birth weight $(\mathrm{BW}<1500 \mathrm{~g})$, and longer stay in hospital $(>14 \mathrm{~d})$ received more KMC.

\section{KMC practice}

Table 2 stratified infants who received KMC by GA and birth weight. $55.3 \%$ of infants below 32 weeks at birth were received KMC, and $56.9 \%$ had a birth weight of < $1500 \mathrm{~g}$. More infants that were immature and with less BW received KMC.

Table 3 presents detailed information of KMC practice by infant's GA at birth. Although babies born at a lower GA and with lower birth weight were more likely to receive $\mathrm{KMC}$ than premature newborns born at a greater GA and heavier birth weight, they were initiated on KMC later and once they had gained weight. Newborns with a lower gestational age at birth started KMC later compared with those whose GA $\geq 32$ weeks at birth. Infants GA $<32$ weeks were initiated KMC later, at day 28.0 median (IQR: 17.0,29.5) and day $23.0(16.0,32.0)$ for infants with GA $<28$ weeks and GA $28-<32$ weeks respectively, compared to day $13.5(8.8,17.3)$ for infants with $\mathrm{GA} \geq 32$ weeks. The weight of smaller GA infants at the time of KMC was still lower: the average weight of all KMC infants were $1809.4 \pm 437.8 \mathrm{~g}$, while infants GA $<28$ weeks had an average weight of $1423.3 \pm 226.8$ g, lower than infants in other GA groups (GA 28- $<32$ weeks $1768.1 \pm 459.2 \mathrm{~g}($ mean \pm SD $)$, GA $32-<34$ weeks $1852.8 \pm 333.6 \mathrm{~g}$, and GA $\geq 34$ weeks $1995.5 \pm 541.4 \mathrm{~g}$ ). KMC duration per session (average hours of KMC received) was $1.7 \pm 1.0 \mathrm{~h}$ on average. The duration per session and frequency of KMC did not vary by gestational age and birth weight groups, although there was a trend toward more frequent $\mathrm{KMC}$ in $\mathrm{GA}<28$ weeks infants.

Figure 3 describes the distribution of accumulated KMC hours for each baby (median, quartile) differed by GA category, with significantly increased greater exposure for infants born at a lower GA $(19 \mathrm{~h}$ for GA $<28$ weeks, and $10 \mathrm{~h}, 6 \mathrm{~h}$, and $8.8 \mathrm{~h}$ for GA 28-32 weeks, 3234 weeks, and 34-37 weeks).

\section{Acceptability of KMC by parents}

A total of 135 questionnaires were sent out to parents providing KMC and 135 parents responded. Parents performed KMC are invited to the survey. Questionnaire respondents could either be the father or mother, though mothers will be selected if both provided KMC. $94.8 \%$ of the parents who participated in the survey stated that KMC was positively accepted by their family members (Fig. 4). Over half of the participants indicated that KMC played a role in relieving anxiety (60.4\%), increasing parental communication with doctors and nurses (57.3\%), and assisted in increasing parental confidence in caring for their preterm babies (69.8\%).

\section{Discussion}

Through the analysis of cross-sectional data collected from eight NICUs in China, we found that, under current circumstances, infants born at a lower gestational age and with a lower birth weight were more likely to receive KMC than premature infants born at a greater gestational age and with a higher birth weight. First, this potentially is due to more and more tiny and sicker preterm infants to be treated in NICU and they need more extra helps besides current standard therapy. Second, medical staff's growing confidence in KMC as a safe and beneficial intervention is important for it could be feasibly provided in NICU. The results of our program emphasis to provide KMC to small/sick babies.

Table 1 Basic characteristics of KMC and no KMC infants

\begin{tabular}{|c|c|c|c|c|}
\hline & KMC group $(N=135)$ & No KMC group $(N=503)$ & $T / x^{2}$ & $P$ \\
\hline Gestational age at birth (week, mean \pm SD) & $31.2 \pm 2.3$ & $33.9 \pm 2.4$ & 10.5 & $<0.01$ \\
\hline Gestational age $<32$ weeks (\%) & 65.2 & 15.9 & 133.3 & $<0.01$ \\
\hline Birth weight < $1500 \mathrm{~g}(\%)$ & 60.7 & 15.3 & 117.4 & $<0.01$ \\
\hline Multiple Births ${ }^{\mathrm{a}}(\%)$ & 38.4 & 37.8 & 0.01 & 0.92 \\
\hline Any breast feeding at discharge (\%) & 78.5 & 72.0 & 2.3 & 0.1 \\
\hline Length of hospital stay $>14 d(\%)$ & 89.6 & 32.3 & 105.9 & $<0.01$ \\
\hline Length of hospital stay $>28 d(\%)$ & 57.3 & 15.3 & 78.5 & $<0.01$ \\
\hline Corrected gestational age at discharge (week, mean \pm SD) & $34.8 \pm 2.4$ & $36.3 \pm 2.1$ & 6.0 & $<0.01$ \\
\hline Weight at discharge $(g$, mean $\pm S D)$ & $1809.4 \pm 436.1$ & $2266.2 \pm 459.5$ & 9.2 & $<0.01$ \\
\hline
\end{tabular}

KMC Kangaroo mother care, SD Standard Deviation; P, $p$-value

${ }^{a}$ include twins and above, $100 \%$ minus percentage of single births 
Table 2 Gestational age, Birth weight distribution of KMC infants

\begin{tabular}{|c|c|c|c|c|c|}
\hline \multirow[t]{2}{*}{ Variable } & & \multirow{2}{*}{$\begin{array}{l}\text { Preterm } \\
\text { infant } \\
\text { discharged }\end{array}$} & \multirow{2}{*}{$\begin{array}{l}\text { Premature } \\
\text { discharged } \\
\text { that } \\
\text { performed } \\
\text { KMC, N(\%) }\end{array}$} & \multirow{2}{*}{\multicolumn{2}{|c|}{ Compared with gestational age $<28$ week or birth weight $<1000 \mathrm{~g}$}} \\
\hline & & & & & \\
\hline Total & & 638 & $135(21.2 \%)$ & & \\
\hline \multirow[t]{4}{*}{ Gestational age (week) } & $<28$ & 24 & $12(50.0 \%)$ & - & - \\
\hline & $28-<32$ & 135 & $76(56.3 \%)$ & 0.3 & 0.6 \\
\hline & $32-<34$ & 155 & $35(22.6 \%)$ & 8.1 & $<0.01$ \\
\hline & $34-<37$ & 324 & $12(3.7 \%)$ & 67.6 & $<0.01$ \\
\hline$x^{2}$ & & & 171.2 & & \\
\hline$P$ & & & $<0.01$ & & \\
\hline \multirow[t]{5}{*}{ Birth weight (g) } & $<1000$ & 27 & $13(48.1 \%)$ & - & - \\
\hline & $1000-<1500$ & 117 & 69 (59.0\%) & 1.0 & 0.3 \\
\hline & $1500-<2000$ & 166 & 41 (24.7\%) & 6.3 & 0.01 \\
\hline & $2000-<2500$ & 191 & $10(5.2 \%)$ & 41.7 & $<0.01$ \\
\hline & $\geq 2500$ & 137 & $3(2.2 \%)$ & 49.0 & $<0.01$ \\
\hline$x^{2}$ & & & 171.0 & & \\
\hline$P$ & & & $<0.01$ & & \\
\hline
\end{tabular}

KMC Kangaroo mother care; \%, KMC premature/preterm infant discharged; $\mathrm{x}^{2}$, Chi-squared test

Preterm infants born at a GA under 28 weeks had more KMC exposure hours during hospitalization than infants born at a higher GA. KMC was well accepted by parents and was perceived as beneficial to reduce anxiety and improve communications between parents and medical staff.

Our study is one of the first to report on the implementation of KMC in China's NICUs following its introduction in selected pilot hospitals. All eight research units included in this study are in tertiary hospitals - including both general teaching hospitals and maternal and child health care hospitals - located in major urban cities across seven different provinces in North, South, West and East China. The number of preterm infants in those NICUs are also relatively large. Our results show that intermittent $\mathrm{KMC}$ is both feasible and acceptable in typical NICU settings across China, and that use of
KMC for relatively small and sick babies was observed. This suggests that our approach of systematic introduction, training and support for implementation may accelerate the use and scale-up of this high impact intervention across China. Despite observed differences in NICU location, bed capacity, patient population and relative differences in KMC implementation, our findings across NICUs showed largely consistent patterns of KMC use, feasibility and acceptability. We believe that combining data across these hospitals provides a valid snapshot of KMC use after introduction and establishment in China's NICUs. Our study differs from previous studies in China that focused on very or extremely low birth weight infants admitted to NICUs in specific hospitals [17]. We looked at KMC use for all newborns admitted to eight participating NICU's, allowing us to identify the newborns. Infants would be prioritized for KMC by

Table 3 KMC initiation and practice by different gestational age group

\begin{tabular}{|c|c|c|c|c|c|c|}
\hline \multirow{2}{*}{$\begin{array}{l}\text { GA } \\
\text { (week) }\end{array}$} & \multirow{2}{*}{$\begin{array}{l}\text { KMC } \\
\text { Number }\end{array}$} & \multicolumn{3}{|l|}{ KMC initiation } & \multicolumn{2}{|l|}{ KMC practice } \\
\hline & & Age $(d)^{*}$ & CGA (week) & Weight (g) & Frequency $(n)^{*}$ & Duration per session (h) \\
\hline Total & 135 & $17.0(11.0,29.0)$ & $34.5 \pm 2.0$ & $1809.4 \pm 437.8$ & $5.0(5.0,7.3)$ & $1.7 \pm 1.0$ \\
\hline$<28$ & 12 & $28.0(17.0,29.5)^{\#}$ & $33.9 \pm 3.8$ & $1423.3 \pm 226.8$ & $13.0(7.5,15.0)$ & $1.7 \pm 0.3$ \\
\hline $28 \sim<32$ & 76 & $23.0(16.0,32.0)^{\#}$ & $33.7 \pm 1.5^{\#}$ & $1768.1 \pm 459.2$ & $5.0(5.0,8.0)$ & $1.8 \pm 1.3$ \\
\hline $32 \sim<34$ & 35 & $14.0(9.0,17.0)$ & $34.9 \pm 1.1$ & $1852.8 \pm 333.6$ & $5.0(4.0,5.0)$ & $1.5 \pm 0.7$ \\
\hline $34 \sim<37$ & 12 & $7.0(5.0,16.5)^{\#}$ & $37.2 \pm 2.3^{\#}$ & $1995.5 \pm 541.4$ & $5.0(5.0,6.0)$ & $1.5 \pm 0.5$ \\
\hline$F / X^{2}$ & & 9.1 & 16.9 & 1.8 & 1.5 & 1.6 \\
\hline P & & $<0.01$ & $<0.01$ & 0.2 & 0.2 & 0.2 \\
\hline
\end{tabular}

KMC Kangaroo mother care, GA Gestational age, $d$ Day, CGA Corrected gestational age, $n$ Number, $h$ Hour; Values are mean \pm SD or median (first quartile, third quartile) $(*) ;$ \# denotes $p<0.05$ while compared with $32 \sim<34$ weeks; 


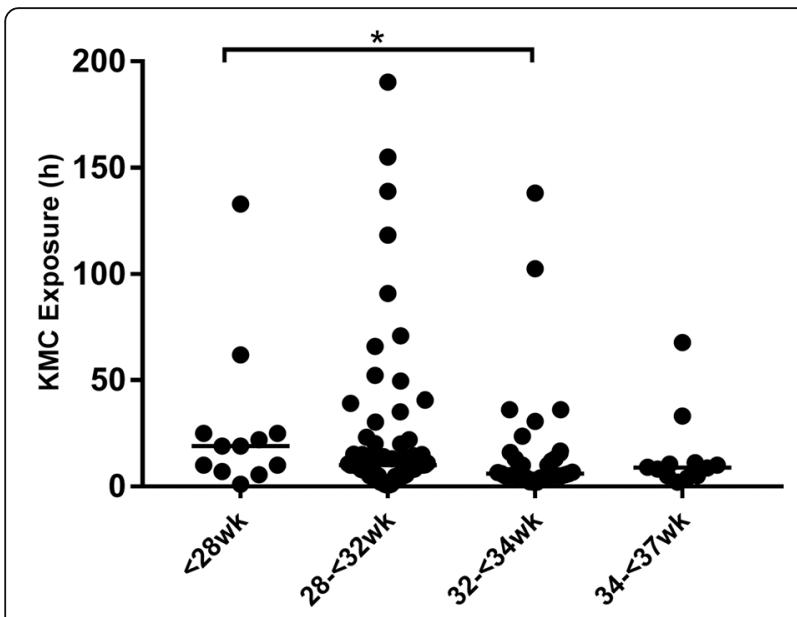

Fig. 3 Total KMC exposure in hours per gestational age preterm infants with $\mathrm{GA}<28 \mathrm{wk}$ being the longest (Median, IQR, $P<0.05$ )

neonatologists and with our geographically representative sample of hospitals our findings are more generalizable for China as a whole.

Some reports of KMC experience in other country settings indicated that $\mathrm{KMC}$ is commonly provided to infants with GA $>32$ weeks or with a birth weight above $1500 \mathrm{~g}$ after becoming clinically stable $[18,19]$. Our study found relatively greater KMC use for smaller and younger GA infants, including many receiving non-invasive and invasive respiratory support. Preterm infants born below 28 weeks GA had a significantly longer average KMC exposure hours compared with those born with a GA above 34 weeks. As tiny babies had more complications, longer stay in hospital, more anxious of their parents, the medical staff prefer providing some additional help. KMC is believed to be able to provide support to preterm infants by medical staffs, so in the situation of limited resource, smaller and sicker infants are more often the beneficiaries of KMC. The training for medical/ nursing staff under the Premature Birth and Preterm Infants Intervention Program in fact emphasized the specific benefits for these more vulnerable babies as long as KMC was carefully used so as to ensure patient safety. In addition to our reported findings, our interviews with medical and nursing staff (not included in the current analysis) consistently found positive views of KMC benefit and safety, with common reports from staff that length of hospitalization among preterm infants was reduced following KMC introduction and increasing implementation. Our speculation appears to be in line with a recently reported national survey of NICU nurses finding increasing KMC implementation experience was associated with substantially greater nurse acceptance and perceived benefits of KMC [20].

Our analysis of the views of parents providing $\mathrm{KMC}$ also indicated that Chinese parents have a high level of acceptance of KMC for preterm infants. KMC was recognized as having positive influence over reducing anxiety and improving communication between parents and medical staff, which was consistent with previous studies e.g. in India [21]. It should be noted that sources of KMC information for parents in China were limited, with most parents receiving their first information on KMC from medical and nursing staff only during NICU hospitalization. While hospital-level promotion should be continued to expose more parents to the concept of KMC, additional advocacy through social media and other channels is needed to promote parental knowledge and increase "demand" for KMC in China.

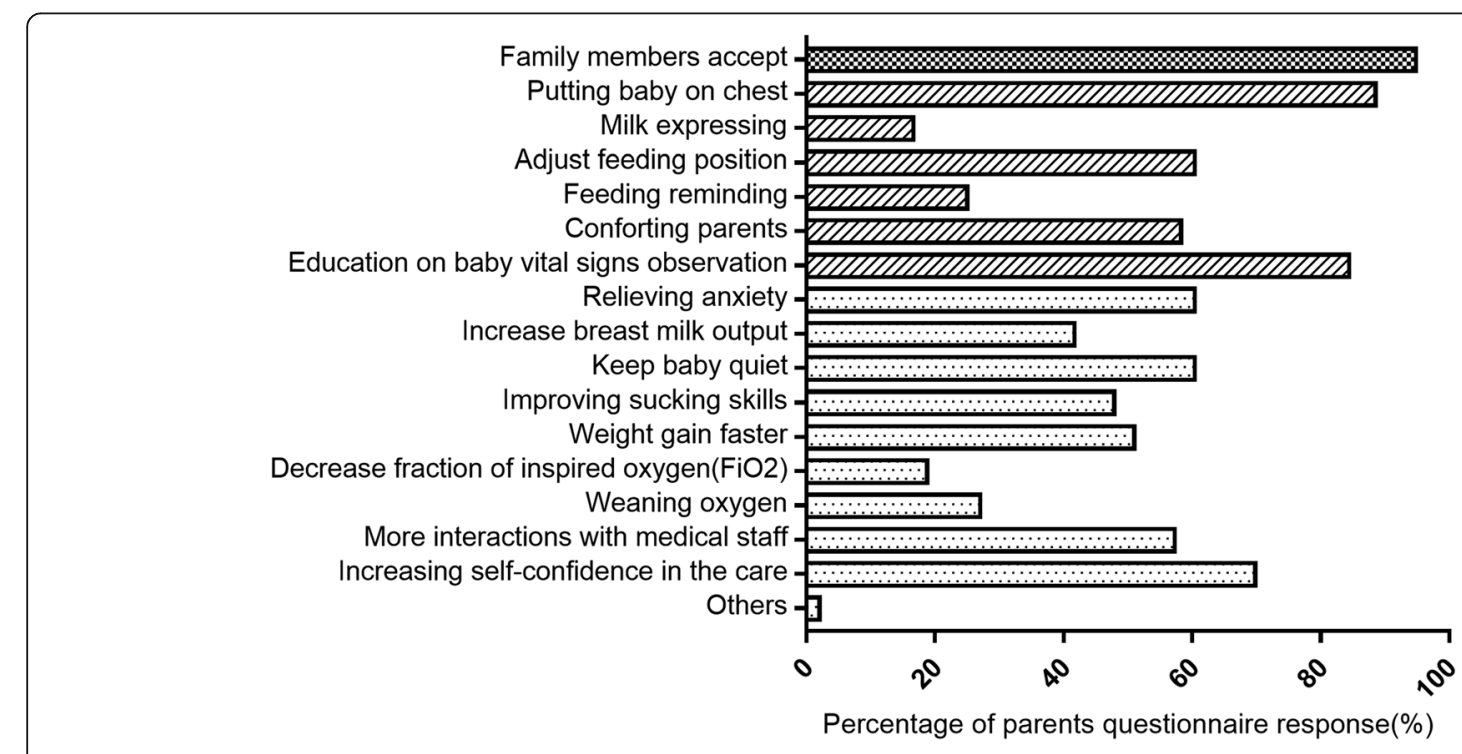

Fig. 4 Parental experience. The bars the represent the proportion of parents who perceived that KMC had a positive effect 
Several limitations should be noted for the current study. To start with, this study is a cross-sectional study which presents the current status of KMC utilization in China's NICUs, thus no casual inference could be made; Secondly, there might be inconsistency among hospitals regarding KMC inclusion, implementation and reporting, as KMC decision-making was largely influenced by hospital's prior experience with KMC, and the perceived advantage and risk of KMC among individual medical staff. Nonetheless, we believe that by aggregating the data across hospitals we have the best-available evidence on KMC utilization in China's NICUs; Thirdly, despite that one of detailed information on the discharge diagnosis which is serious infection collected, we cannot specify whether it occurred during or before the KMC sessions, which is another limitation for the current analysis.

\section{Conclusion}

In this cross-sectional study, we analyzed and reported on the current status of intermittent KMC use in selected Chinese NICUs after prior staff exposure to advocacy and training, and the sharing of experiences among participating hospital staff. Preterm infants selected by neonatologists as eligible for $\mathrm{KMC}$ were found to be those who were more premature. KMC was highly accepted by parents of preterm infants. Considering safety issues like the low risk of infections, we recommend that KMC guidelines and protocols appropriate for China's NICU settings should be developed, endorsed and implemented to enable nation-wide scale up of KMC.

\section{Supplementary information}

Supplementary information accompanies this paper at https://doi.org/10. 1186/s12887-020-02153-2.

Additional file 1. Survey questionnaire for mothers who performed KMC (filled prior to discharge).

\section{Abbreviations}

CGA: Corrected gestational age; GA: Gestational age; KMC: Kangaroo mother care; NICU: Neonatal intensive care unit

\section{Acknowledgements}

We thank all parents and infants, all participants in KMC delivery and data collection, all international and national professional experts for technical support. Special thanks to program support from Save the Children. Also cordially thank Sarah Williams, Steve Wall, Greta Wetzel, Zhao Li, Lin Zhang, Ying Cao, and Yingxi Zhao, for their insightful discussions in this work.

\section{Authors' contributions}

QF and GZ conceived of and led the study design; QF and XL coordinated data collection, analysis and wrote the first draft of the manuscript; $Z \mathrm{~L}, \mathrm{XC}$, BC, SJ, CYY, QL and CZY contributed to data collection, training and program implementation. The authors have read and approved the manuscript.

\section{Funding}

This study was funded by Infants Program of China (No. 34400038) from the Save the Children International. The funding body has role in designing the study, analysis, and interpretation of data and in writing the manuscript.

\section{Availability of data and materials}

The datasets used during the current study are available from the corresponding author on reasonable request.

\section{Ethics approval and consent to participate}

Ethical approval was obtained from Peking University First Hospital Biomedical Research Ethics Committee [(2018) scientific research no. (13)]. Written consent was obtained from the parents of newborns who received KMC.

\section{Consent for publication}

Not applicable; no individual person's personal information is included in this study.

\section{Competing interests}

The authors declare that they have no conflict of interest.

\section{Author details}

${ }^{1}$ Neonatal Intensive Care Unit, Department of Pediatrics, Peking University First Hospital, Beijing, MM, China. ${ }^{2}$ Department of Neonatology, Northwest Women's and Children's Hospital, Shanxi, MD, China. ${ }^{3}$ Department of Neonatology, Nanjing Maternity and Child Health Care Hospital, Jiangsu, MM, China. ${ }^{4}$ Department of Neonatology, Hunan Provincial Maternal and Child Health Care Hospital, Hunan, China. ${ }^{5}$ Department of Neonatology, Xiangya Hospital Central South University, Hunan, MD, China. ${ }^{6}$ Department of Neonatology, Fujian Provincial Maternity and Children's Hospital, Fujian, China. ${ }^{7}$ Department of Neonatology, Women \& Children's Health Care Hospital of Linyi, Shandong, China. ${ }^{8}$ Department of Neonatal, Shenzhen Maternity \& Child Healthcare Hospital, Shenzhen, MD, China. 'Department of Obstetrics and Gynecology, Peking University First Hospital, Beijing, MB, China.

Received: 19 January 2020 Accepted: 19 May 2020

Published online: 29 May 2020

\section{References}

1. World Bank. Mortality rate, under-5 (per 1,000 live-births) - China. Retrievd from https://data.worldbank.org/indicator/SH.DYN.MORT?locations= CN\&view=chart

2. HNN. China - Health Newborn Network. Retrieved from https://www. healthynewbornnetwork.org/country/china.

3. Chawanpaiboon S, Vogel JP, Moller AB, Lumbiganon P, Petzold M, Hogan D, et al. Global, regional, and national estimates of levels of preterm birth in 2014: a systematic review and modelling analysis. Lancet Glob Health. 2019; 7(1):e37-46.

4. Bergman NJ, Jurisoo LA. The 'kangaroo-method' for treating low birth weight babies in a developing country. Trop Doct. 1994;24(2):57-60.

5. WHO Guidelines Approved by the Guidelines Review Committee. WHO recommendations on interventions to improve preterm birth outcomes. Geneva: World Health Organization Copyright (c) World Health Organization 2015; 2015.

6. Charpak N, Ruiz-Pelaez JG, de Figueroa CZ, Charpak Y. Kangaroo mother versus traditional care for newborn infants </=2000 grams: a randomized, controlled trial. Pediatr. 1997;100(4):682-8.

7. Parmar VR, Kumar A, Kaur R, Parmar S, Kaur D, Basu S, et al. Experience with kangaroo mother care in a neonatal intensive care unit (NICU) in Chandigarh, India. Indian J Pediatr. 2009;76(1):25-8.

8. Morelius E, Ortenstrand A, Theodorsson E, Frostell A. A randomised trial of continuous skin-to-skin contact after preterm birth and the effects on salivary cortisol, parental stress, depression, and breastfeeding. Early Hum Dev. 2015:91(1):63-70.

9. Parsa P, Karimi S, Basiri B, Roshanaei G. The effect of kangaroo mother care on physiological parameters of premature infants in Hamadan City, Iran. Pan Afr Med J. 2018;30:89. 
10. Feldman R, Rosenthal Z, Eidelman Al. Maternal-preterm skin-to-skin contact enhances child physiologic organization and cognitive control across the first 10 years of life. Biol Psychiatry. 2014;75(1):56-64.

11. He L, TAN YJ, HEI MY. Effect of family integrated care in neonatal intensive care unit on maternal stressfulness of hospitalized preterm infants: a self comparison study. Chin J Evid Based Pediatr. 2015;10(6):409-13.

12. Deng $\mathrm{Q}, \mathrm{Li} \mathrm{Q}$, Wang $\mathrm{H}$, Sun $H$, Xu X. Early father-infant skin-to-skin contact and its effect on the neurodevelopmental outcomes of moderately preterm infants in China: study protocol for a randomized controlled trial. Trials. 2018;19(1):701.

13. Mellis C. Kangaroo mother care and neonatal outcomes: a meta-analysis. J Paediatr Child Health. 2016;52(5):579.

14. Casper C, Sarapuk I, Pavlyshyn H. Regular and prolonged skin-to-skin contact improves short-term outcomes for very preterm infants: a dosedependent intervention. Arch Pediatr. 2018:25(8):469-75.

15. Deng $Q$, Zhang $Y, L i Q$, Wang $H, X u X$. Factors that have an impact on knowledge, attitude and practice related to kangaroo care: national survey study among neonatal nurses. J Clin Nurs. 2018;27(21-22):4100-11.

16. Chan G, Bergelson I, Smith ER, Skotnes T, Wall S. Barriers and enablers of kangaroo mother care implementation from a health systems perspective: a systematic review. Health Policy Plan. 2017;32(10):1466-75.

17. Hei MY, Gao XY, Li ZK, Zhang QS, Li J, Xia SW, et al. Breastfeeding outcomes in family integrated care model in neonatal intensive care units. Chin J Neonatol. 2018;33(1):27-33.

18. Samra NM, Taweel AE, Cadwell K. Effect of intermittent kangaroo mother care on weight gain of low birth weight neonates with delayed weight gain. J Perinat Educ. 2013;22(4):194-200

19. Charpak N, Ruiz-Pelaez JG, de Figueroa CZ, Charpak Y. A randomized, controlled trial of kangaroo mother care: results of follow-up at 1 year of corrected age. Pediatrics. 2001;108(5):1072-9.

20. Zhang Y, Deng Q, Zhu B, Li Q, Wang F, Wang H, et al. Neonatal intensive care nurses' knowledge and beliefs regarding kangaroo care in China: a national survey. BMJ Open. 2018;8(8):e021740.

21. Gathwala G, Singh B, Singh J. Effect of kangaroo mother care on physical growth, breastfeeding and its acceptability. Trop Doct. 2010;40(4):199-202.

\section{Publisher's Note}

Springer Nature remains neutral with regard to jurisdictional claims in published maps and institutional affiliations.

Ready to submit your research? Choose BMC and benefit from:

- fast, convenient online submission

- thorough peer review by experienced researchers in your field

- rapid publication on acceptance

- support for research data, including large and complex data types

- gold Open Access which fosters wider collaboration and increased citations

- maximum visibility for your research: over $100 \mathrm{M}$ website views per year

At $\mathrm{BMC}$, research is always in progress.

Learn more biomedcentral.com/submissions 